\title{
Rare Etiology of Renal Failure in a 25-Year-Old Caucasian Man: Fabry Disease With a Novel Mutation of GLA Gene
}

\author{
Salem Gaballa ${ }^{1}$, Avan AlJaf ${ }^{1}$, Jane Lindsay ${ }^{1}$, Kashyap Patel ${ }^{1}$, Kyaw M. Hlaing ${ }^{1}$ \\ 1. Internal Medicine, LewisGale Medical Center, Salem, USA
}

Corresponding author: Salem Gaballa, salem.taher90@gmail.com

\begin{abstract}
Fabry disease (FD) is an X-linked recessive lysosomal storage disease caused by a mutation of the galactosidase alpha (GLA) gene, leading to deficiency of $\alpha$-galactosidase A (alpha-Gal A). This deficiency results in a progressive, multiorgan accumulation of glycolipids, most notably globotriaosylceramide (Gb3), leading to multiorgan failure and subsequently premature death. Gb3 accumulation in the podocytes, epithelial, and mesangial cells of the glomeruli results in progressive renal disease and eventually renal failure and hemodialysis (HD). There are two types of FD: early-onset classical type 1 and late-onset type 2. Although nearly a thousand mutations of the GLA gene have been identified, the majority of them are of unknown significance. Herein we report the case of a 25 -year-old Caucasian male with no significant medical history who presented with peripheral neuropathy and end-stage renal failure, requiring HD. He was diagnosed with FD based on the electron microscopy findings of renal biopsy and severely reduced alphaGal A activity ( $<0.4 \mathrm{nmol} / \mathrm{mL} /$ hour). A novel mutation of c.281G $>T$; p.Cys 94 Phe was identified. On discharge from our facility, he was referred to a renal transplant center and genetic counseling.
\end{abstract}

Received 07/03/2020

Review began $07 / 03 / 2020$ Review ended 07/04/2020 Published 07/11/2020

(c) Copyright 2020

Gaballa et al. This is an open access article distributed under the terms of the Creative Commons Attribution License CC-BY 4.0., which permits unrestricted use, distribution, and reproduction in any medium, provided the original author and source are credited.
Categories: Genetics, Internal Medicine, Nephrology

Keywords: fabry's disease, painful neuropathy, renal failure, lysosomal storage disease, $\alpha$-galactosidase a activity, enzyme replacement therapy

\section{Introduction}

Fabry disease (FD) is the second most prevalent lysosomal storage disorder after Gaucher disease [1]. It is an $\mathrm{X}$-linked inherited mutation of the galactosidase alpha (GLA) gene of the $\mathrm{X}$ chromosome [2]. These mutations result in the absence or deficiency of $\alpha$-galactosidase A (alpha-Gal A) enzyme, which catalyzes the hydrolytic cleavage of the terminal galactose from globotriaosylceramide (Gb3), leading to multiorgan glycosphingolipid accumulations. The prevalence of classic FD is estimated to range from 1:8,454 to $1: 117,000$ in males, and the disease is seen across all ethnic and racial groups [3]. Diagnosis of FD is challenging; therefore, if physical and clinical examination raises a suspicion of FD, biochemical and/or genetic tests could be considered to confirm the diagnosis [4].

\section{Case Presentation}

A 25-year-old male with no past medical history was brought to the emergency department with complaints of tingling and severe burning sensation in the hands and feet for several days. He endorsed associated nausea and non-bilious emesis, poor appetite, and mental fogginess. He also noted decreased urine output, without any dysuria, hematuria, or lower back pain. He denied any chest pain, palpitation, shortness of breath, abdominal pain, diarrhea, profuse sweating, or heat or cold intolerance. He denied a history of smoking cigarettes or drinking alcohol. He did endorse a family history of FD in his aunt. Physical examination was remarkable for pale conjunctiva, angiokeratoma of fingertips (Figure 1), and asterixis. His vital signs were only remarkable for elevated blood pressure of 180/100. 


\section{Cureus}

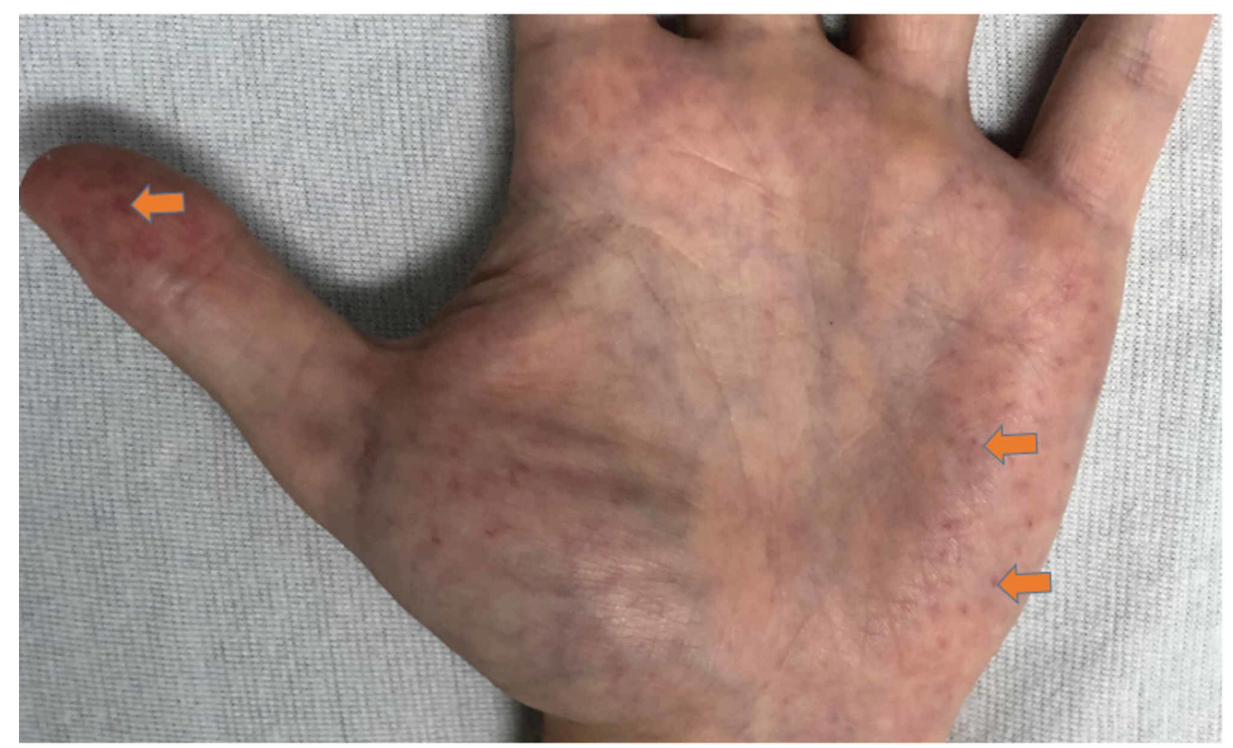

FIGURE 1: Angiokeratoma of the distal thumb and palm.

Complete blood count (CBC) revealed white blood cells of 9.16 cells/mcL (normal range: 4,50011,000 cells/mcL), hemoglobin (Hgb) of $7.9 \mathrm{~g} / \mathrm{dL}$ (normal range: $14-16 \mathrm{~g} / \mathrm{dL}$ ), hematocrit (Hct) of $22.6 \%$ (normal range for adult males: 40\%-50.3\%), and platelets of 215 cells/mcL (normal range: 150,000-400,000 cells/mcL). Basic metabolic profile (BMP) revealed sodium of $137 \mathrm{mEq} / \mathrm{L}$ (normal range: $135-145 \mathrm{mEq} / \mathrm{L}$ ), potassium of $4.8 \mathrm{mEq} / \mathrm{L}$ (normal range: $3.5-5.2 \mathrm{mEq} / \mathrm{L}$ ), chloride of $103 \mathrm{mEq} / \mathrm{L}$ (normal range: 96-106 $\mathrm{mEq} / \mathrm{L}$ ), carbon dioxide of $20 \mathrm{mEq} / \mathrm{L}$ (normal range: $23-29 \mathrm{mEq} / \mathrm{L}$ ), blood urea nitrogen of $122 \mathrm{mg} / \mathrm{dL}$ (normal range: 6-20 mg/dL), creatinine of $21 \mathrm{mg} / \mathrm{dL}$ (normal range: $0.8-1.2 \mathrm{mg} / \mathrm{dL}$ ), glomerular filtration rate (GFR) of $2.7 \mathrm{~mL} /$ minute/1.73 $\mathrm{m}^{2}$ (normal range: $90-120 \mathrm{~mL} /$ minute $/ 1.73 \mathrm{~m}^{2}$ ), calcium of $7.1 \mathrm{mg} / \mathrm{dL}$ (normal range: 8.6 $10.3 \mathrm{mg} / \mathrm{dL}$ ), phosphate $9 \mathrm{mg} / \mathrm{dL}$ (normal range: $2.5-4.5 \mathrm{mg} / \mathrm{dL}$ ), and albumin 2.9 of g/dL (normal range: 3.4$5.4 \mathrm{~g} / \mathrm{dL}$ ). Liver function panel was within the normal limits. Troponin was $<0.015 \mathrm{ng} / \mathrm{mL}$ (normal range: 0 $0.015 \mathrm{ng} / \mathrm{mL})$.

Urinalysis showed nephrotic range proteinuria (urine protein/creatinine ratio of 5.07), and microscopic hematuria ( $>10$ red blood cell [RBC], few RBC casts). Erythrocyte sedimentation rate (ESR) was $89 \mathrm{~mm}$ /hour (normal range: 0-26 mm/hour). Vitamin B12 was $556 \mathrm{pg} / \mathrm{mL}$ (normal range: $254-1,320 \mathrm{pg} / \mathrm{mL}$ ), vitamin D 25hydroxy was $26.6 \mathrm{ng} / \mathrm{mL}$ (normal range: $30-100 \mathrm{ng} / \mathrm{mL}$ ), and intact parathyroid hormone was $223.3 \mathrm{pg} / \mathrm{mL}$ (normal range: $18.5-88 \mathrm{pg} / \mathrm{mL}$ ). Iron studies revealed iron of $89 \mathrm{mcg} / \mathrm{dL}$ (normal range: $60-170 \mathrm{mcg} / \mathrm{dL}$ ), total iron binding capacity of $194 \mathrm{mcg} / \mathrm{dL}$ (normal range: $240-450 \mathrm{mcg} / \mathrm{dL}$ ), transferrin saturation of $45.9 \%$ (normal range: $20 \%-50 \%$ ), and ferritin of $210 \mathrm{ng} / \mathrm{mL}$ (normal range: $24-336 \mathrm{ng} / \mathrm{mL}$ ).

Electrocardiogram (ECG) showed normal sinus rhythm with left ventricular hypertrophy (LVH) (Figure 2). Computed tomography (CT) of the abdomen and pelvis without intravenous contrast (Figure 3) showed bilateral renal atrophy, without any evidence of hydronephrosis, pyelonephritis, renal mass, or vascular abnormality. Viral hepatitis panel, HIV panel, and toxicology were negative. The antinuclear antibody (ANA) screen, cytoplasmic and perinuclear antineutrophil cytoplasmic antibodies (P-ANCA and C-ANCA), complement levels, and antiglomerular basement membrane (anti-GBM) antibody were all negative. Nephrology service was consulted, and the patient was started on HD due to uremic neuropathy and encephalopathy. Due to the patient's family history of FD, severe neuropathy, and nephrotic range of proteinuria, the genetic testing, alpha-Gal A activity test, and renal biopsy were performed. The biopsy was limited, with not enough glomeruli for light microscopy (LM) or immunofluorescence microscopy, but electron microscopy (EM) showed numerous electron-dense myelin bodies in the endothelial cell cytoplasm of a glomerular capillary loop, multilamellated myelin bodies (zebra bodies) within the cytoplasm of a tubular epithelial cell, and endothelial cells (Figures 4, 5). Echocardiogram (ECHO) showed mild-tomoderate LVH (Figure ๑) and mild pulmonary hypertension with pulmonary artery systolic pressure of 44 $\mathrm{mm} / \mathrm{hg}$ with an estimated ejection fraction of $55-60 \%$. Alpha-Gal A activity was significantly reduced, $<0.4$ $\mathrm{nmol} /$ hour/mg protein (reference range: 42.1 to $112.9 \mathrm{nmol} / \mathrm{hour} / \mathrm{mg}$ protein), which confirm the diagnosis of FD. GAL gene sequencing revealed a novel mutation of c.281G>T; p.Cys94Phe.The patient's peripheral neuropathy and encephalopathy continued to improve on HD, and his blood pressure improved with hydralazine and amlodipine. He was discharged home with continued outpatient HD, with referral to the renal transplant center along with genetic counseling. 


\section{Cureus}

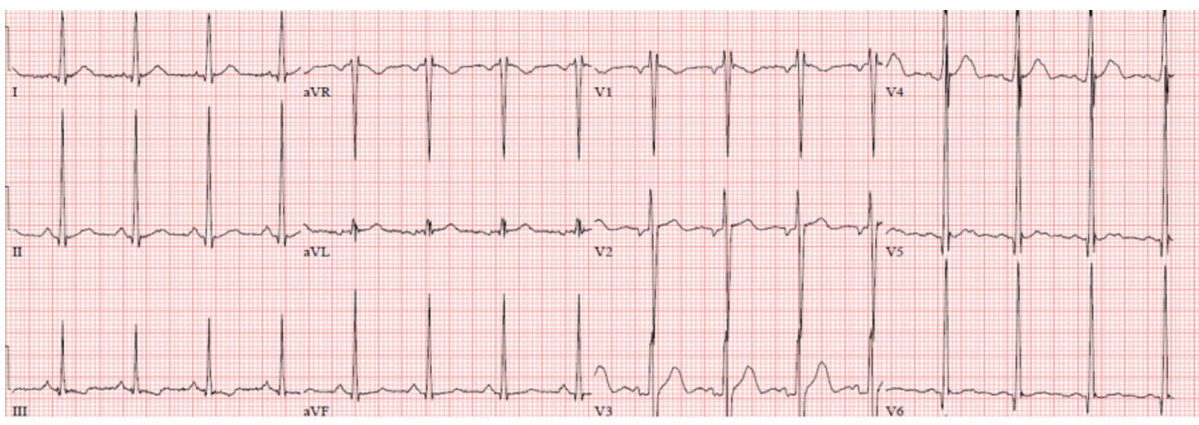

FIGURE 2: ECG showed normal sinus rhythm, with normal axis and intervals, and LVH.

ECG, electrocardiogram; LVH, left ventricular hypertrophy

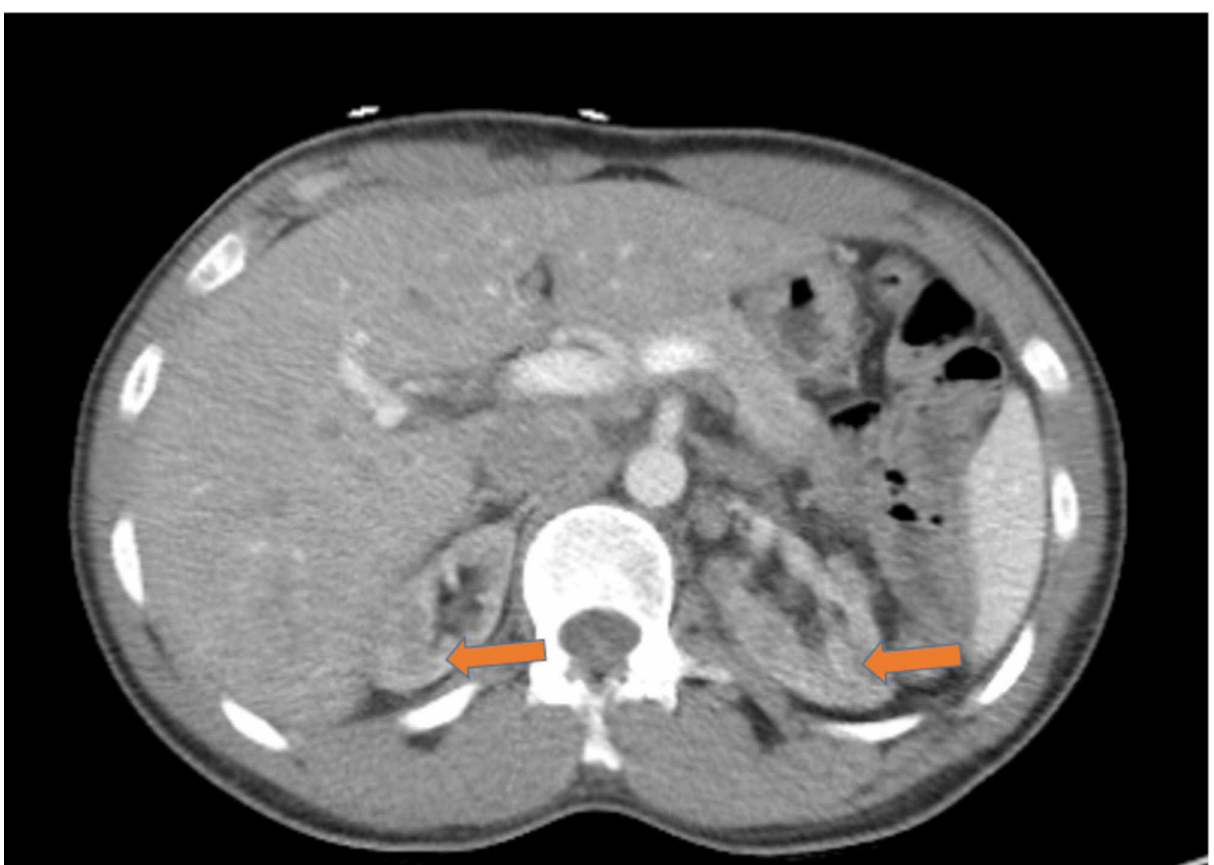

FIGURE 3: CT of the abdomen and pelvis without intravenous contrast in an axial view showing bilateral renal atrophy, without any evidence of hydronephrosis, pyelonephritis, renal mass, or vascular abnormality.

$\mathrm{CT}$, computed tomography

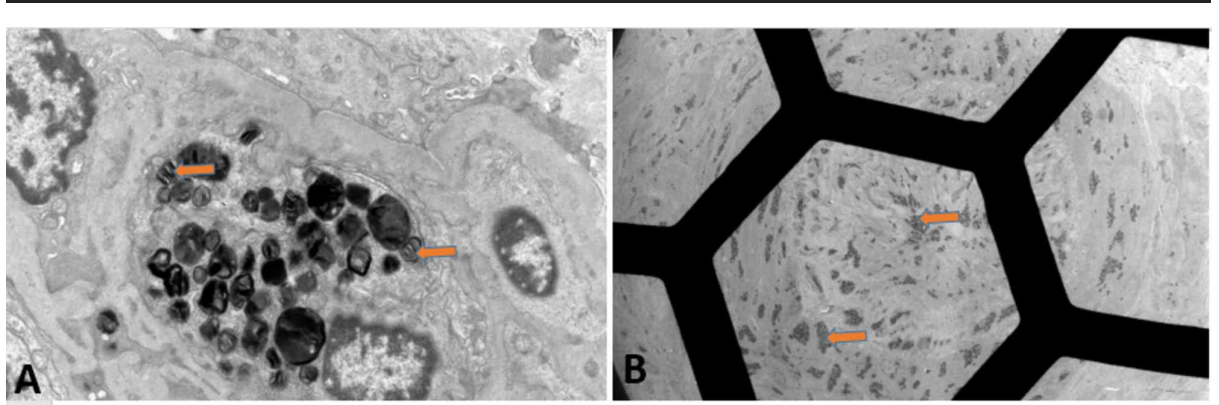

FIGURE 4: EM of renal biopsy showing (A) numerous electron-dense myelin bodies in the endothelial cell cytoplasm of a glomerular capillary loop and (B) artery with myelin bodies visible within smooth muscle and 


\section{Cureus}

endothelial cells.

EM, electron microscopy

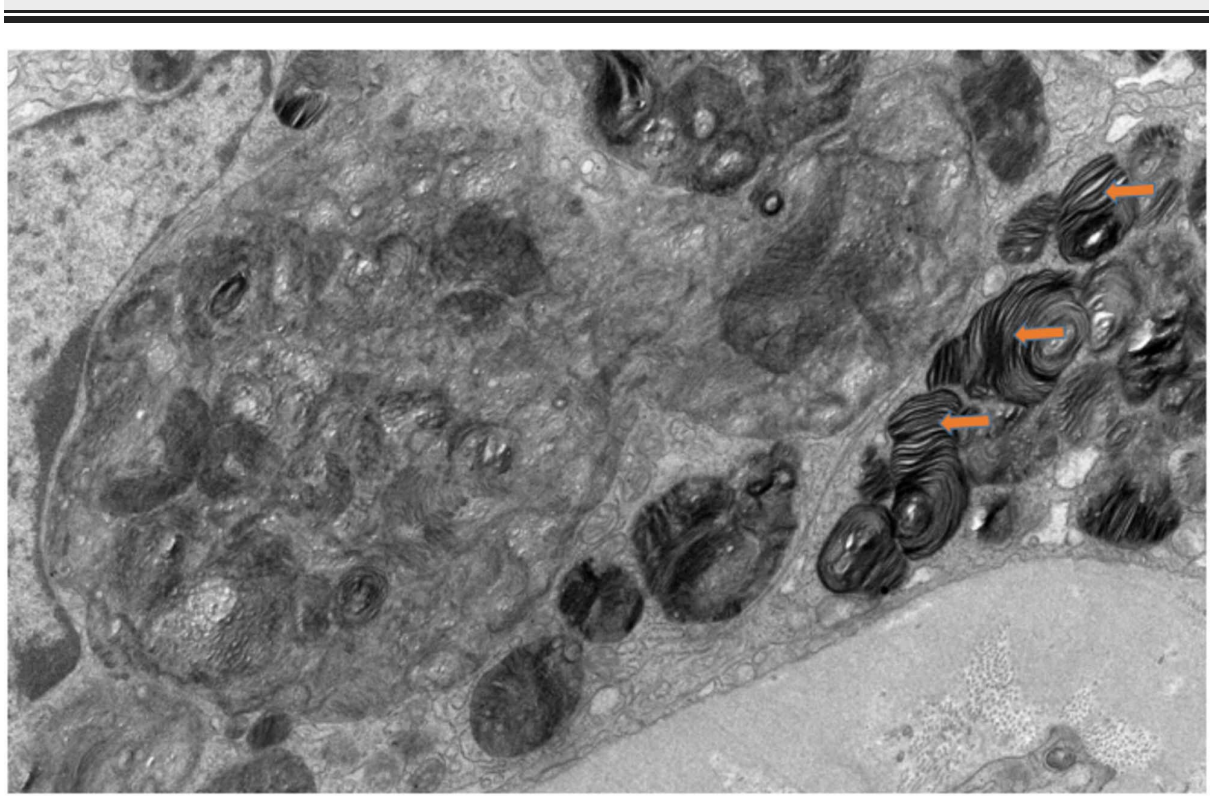

FIGURE 5: EM showing multilamellated myelin bodies (zebra bodies) within the cytoplasm of a tubular epithelial cell.

EM, electron microscopy
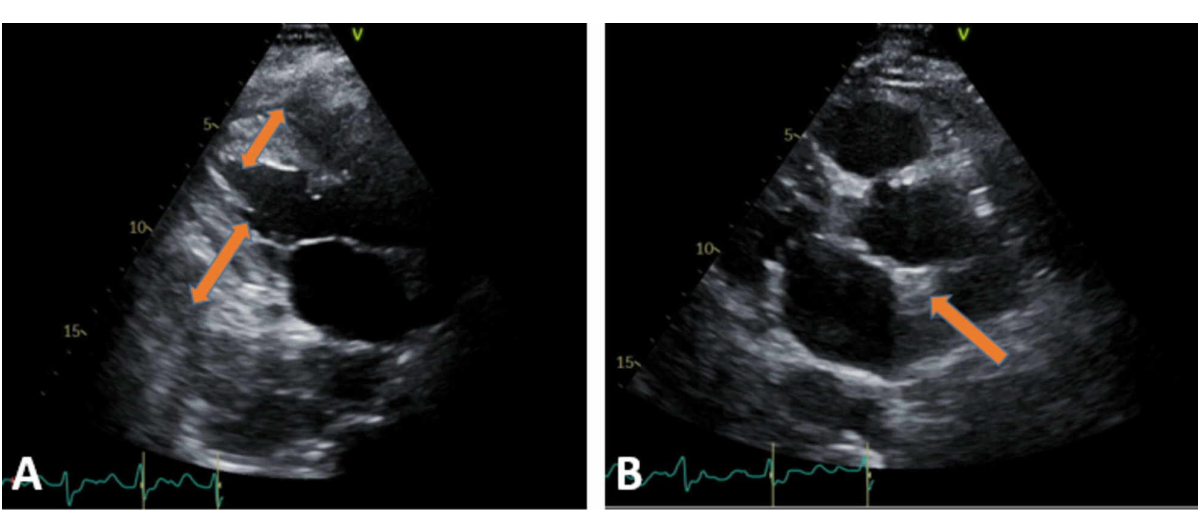

FIGURE 6: ECHO: (A) TTE in parasternal long-axis view demonstrating concentric LVH and thick IVS, as pointed by the orange arrow, and (B) TTE subcostal view demonstrating concentric LVH.

ECHO, echocardiogram; TTE, transthoracic echocardiogram; LVH, left ventricular hypertrophy; IVS, interventricular septum

\section{Discussion}

The pathophysiology of FD is the absence of significant alpha-Gal A activity that results in the Gb3 accumulation in various cells and tissues, causing cytotoxic, pro-inflammatory, and pro-fibrotic effects [5]. Accumulation of Gb3 is particularly prominent in the vascular endothelium (at levels up to 460 -fold higher than normal), vascular smooth muscle cells, and pericytes [6]. The deposition of Gb3 in these cells may lead to the death of smooth muscle cells, vascular occlusion, ischemia, and infarction. There is a relationship between alpha-Gal A enzyme activity and disease symptoms. Mutations that result in little to no alpha-Gal A activity cause the classic early-onset type 1 Fabry phenotype. Those that result in residual alpha-Gal A activity cause the atypical later-onset type 2 phenotype [6]. The prevalence of classic FD is estimated to range from 1:8454 to 1:117,000 in males, and probably underestimated due to the nonspecific 
Type $1 \mathrm{FD}$ is the most severe clinical phenotype and occurs predominantly in males, although some heterozygous females have a severe phenotype that resembles classic FD in males [8]. Males with type 1 FD have little or no functional alpha-Gal A enzyme activity ( $<1 \%$ of the normal mean) [8]. Clinical manifestations of classic FD begin in childhood or adolescence and include severe neuropathic or limb pain (acroparesthesias), which occur in more than $75 \%$ of patients. Telangiectasias, angiokeratomas, gastrointestinal dysfunction, and corneal opacities (cornea verticillata) are also seen. In adulthood, there are progressive renal manifestations such as proteinuria, or unexplained renal insufficiency, and progressive cardiac and cerebrovascular manifestation such as concentric LVH, heart failure, coronary artery disease, conduction abnormalities, transient ischemic attacks, and ischemic strokes [9]. Other less common and nonspecific manifestations include exercise intolerance, anhidrosis or hyperhidrosis, and hearing loss [9].

Type 2 FD usually presents later in life (third to seventh decades of life) than those with the classic form of the disease. They have residual alpha-Gal A activity (between $2 \%$ and $30 \%$ of the normal mean) and may not have Gb3 accumulation in capillaries and small blood vessels [10]. Most do not display the classical features of FD, and their disease is typically dominated by a particular organ system, most commonly the heart. The diagnosis is often made incidentally during evaluation of unexplained LVH, heart failure, arrhythmias, proteinuria, kidney failure, or cryptogenic stroke [11].

Renal manifestations are common among males and, to a lesser extent, female patients with FD. Proteinuria, one of the initial renal findings, occurs in approximately $50 \%$ of untreated males with classic FD by the age of 35 years [12]. The prevalence of proteinuria in males increases with age, reaching approximately $90 \%$ by the age of 50 years [12]. Approximately $30 \%$ to $35 \%$ of females with FD have overt proteinuria (>300 $\mathrm{mg} / \mathrm{day}$ ), with an onset that is typically later than that in males. A significant fraction of patients develop chronic kidney disease and eventually end-stage renal disease (ESRD) [12]. The prevalence of FD in dialysis populations has been examined in several screening studies. Random screening has identified less than $1 \%$ of hemodialysis (HD) patients as having FD. According to Tanaka et al., a screening of alpha-Gal A activity in $696 \mathrm{HD}$ patients (295 females) found only four males and one female $(0.7 \%)$ to have FD [13].

The pathophysiological changes of Fabry nephropathy are due to Gb3 accumulation in the glomeruli (podocytes, endothelial, mesangial, and parietal epithelial cells), distal tubular cells, and vascular smooth muscle cells. The predilection for podocytes may explain the early renal manifestations of proteinuria. In children with FD, podocyte $\mathrm{Gb3}$ accumulation increased with age and was strongly associated with podocyte foot process width and degree of proteinuria, implicating glomerular involvement and early-onset proteinuria observed in this disease [14]. According to Fall et al., podocyte loss in the urine (podocyturia) is increased in patients with FD and correlates with the clinical severity of kidney disease, suggesting that podocyte loss may be important in the progression of Fabry nephropathy [15]. Kidney biopsy, although not required, may be helpful in establishing the diagnosis of FD. Occasionally, the diagnosis of FD is made incidentally when a kidney biopsy is obtained to evaluate the cause of proteinuria and/or decreased kidney function.

Abnormal kidney biopsy findings by LM and EM are characteristic in FD. LM shows foamy vacuolization mostly in the visceral glomerular epithelial cells (podocytes) and distal tubular epithelial cells. This is consistent with the described pattern of glycolipid accumulation (Figure 7) [16]. In addition, renal arteries and arterioles show smooth muscle cell Gb3 accumulation as well as smooth muscle degenerative changes representing the death of smooth muscle cells. On EM, deposits of Gb3 appear primarily within enlarged secondary lysosomes as multilamellated membrane structures called zebra bodies (Figure 8 ) [16]. 


\section{Cureus}
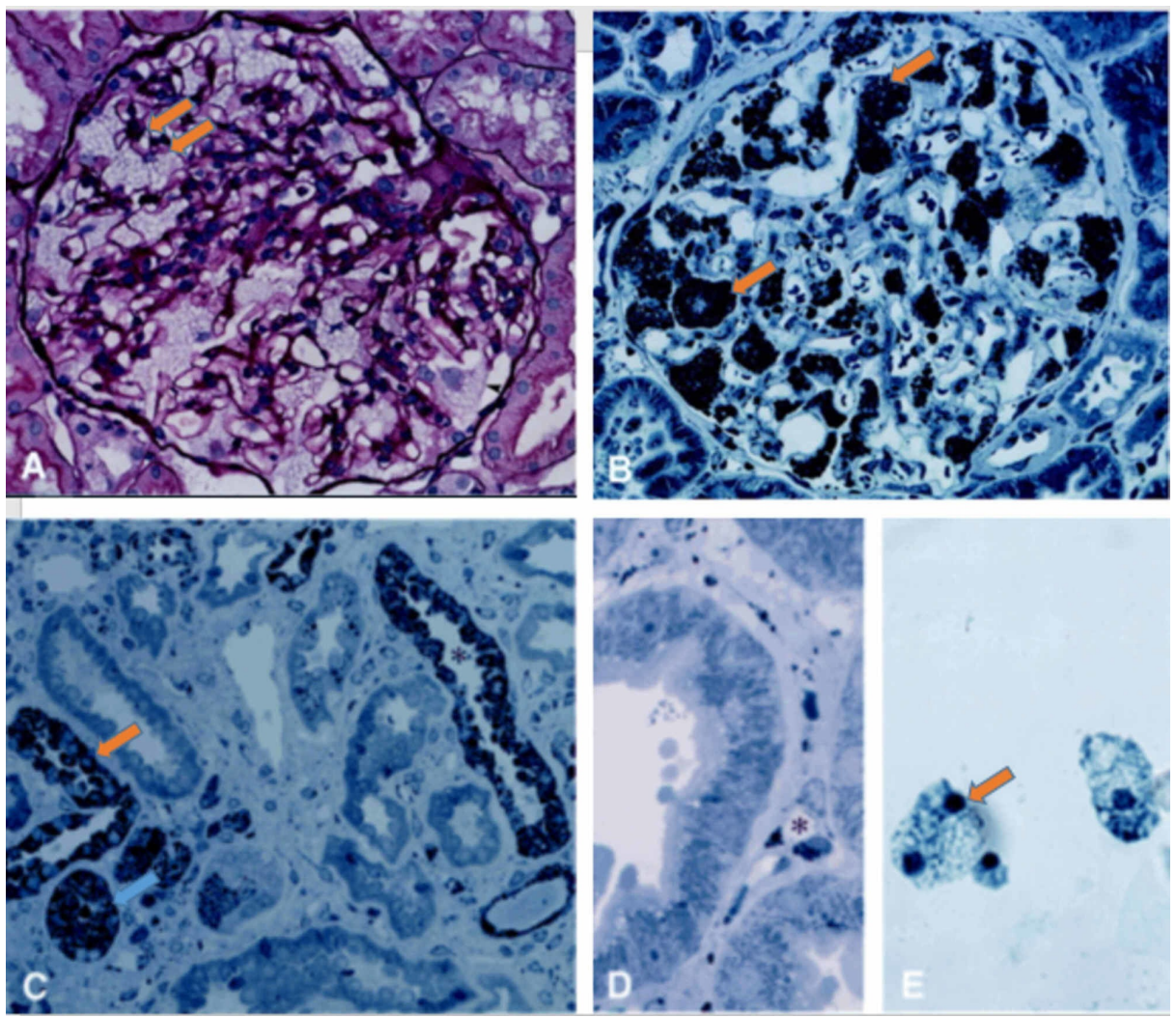

FIGURE 7: LM of renal biopsy of FD.

(A) Glomerulus showing a glycolipids accumulation in the podocytes (arrowhead), with a mesangial widening (PAS stain; magnification, $\times 80$ ). (B) Embedded renal tissue showing glycolipid accumulation in glomerular podocytes (arrowhead; toluidine blue stain; magnification, $\times 80$ ). (C) Embedded renal tissue showing glycolipid accumulation in distal tubules (asterisk), with sparing of proximal tubules, and interstitial fibrosis (toluidine blue stain; magnification, $\times 80$ ). (D) Glycolipid accumulation in the endothelial cells of peritubular capillaries (asterisk; toluidine blue stain; magnification, $\times 200$ ). (E) Urine demonstrating vacuolated epithelial cells (Papanicolaou stain; magnification, $\times 160$ ).

Figure modified with permission from Branton et al [16]. Image reproduction approved by Wolters Kluwer.

LM, light microscopy; FD, Fabry disease 


\section{Cureus}
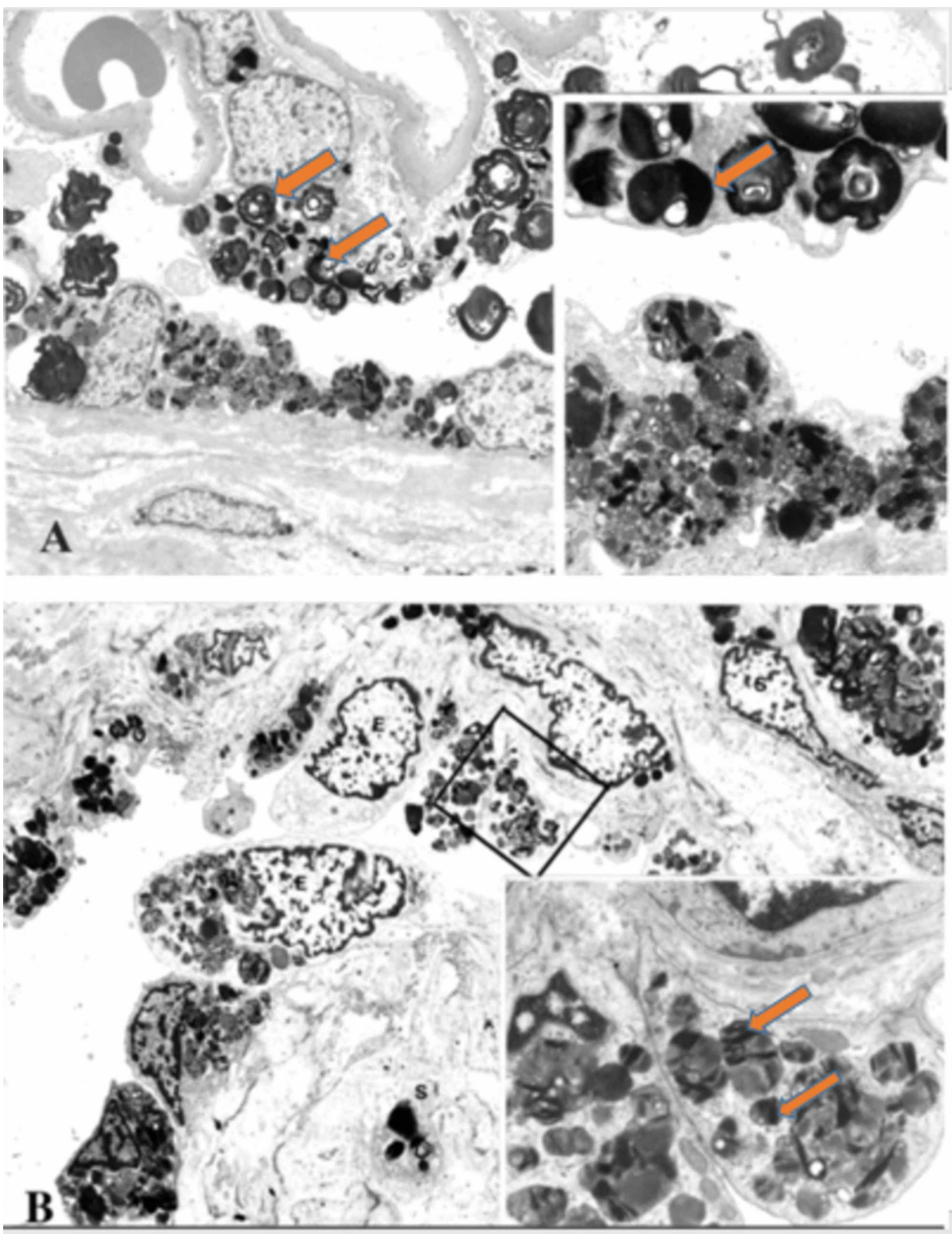

FIGURE 8: (A) EM of renal biopsy of Fabry disease showing the accumulation of glycolipids within the lysosomes of podocytes and glomerular parietal epithelial cells, with a focal foot process effacement. There is accumulation of glycolipid in the urinary space and a thickened Bowman's capsule (magnification, $\times 3760$ ). Higher magnification showing enlarged lysosomes within the podocyte and a combination of multilamellated membrane structures (zebra bodies) and small vesicles in the glomerular parietal epithelium (magnification, $\times 11,200$ ). (B) EM showing the lysosomal storage within endothelial cells of a small artery and smooth muscle cells (magnification, $\times 3800$ ). Higher magnification of the endothelial cell cytoplasm showing enlarged secondary lysosomes filled with glycolipids particles of a heterogeneous appearance (magnification, $\times 11,200$ ).

EM, electron microscopy

These inclusions, including concentric layers with a periodicity of 3.5-5 nm and an onion skin appearance, are considered a hallmark of glycolipid storage disorders. Inclusions may be present in all glomerular cell 
types (most predominantly podocytes), arteriolar smooth muscle cells, and tubules (mostly distal tubules). There is an inverse correlation between the alpha-Gal A activity and renal Gb3 content; thus, glomerular and tubulointerstitial changes and kidney function is worse in patients with undetectable alpha-Gal A activity compared with those with greater than $1 \%$ of normal activity [17].

The treatment and prognosis of patients with FD have tremendously changed over the years due to enzyme replacement therapy (ERT), migalastat, HD, and renal transplantation. The main treatment of FD has focused on replacing the missing or deficient enzyme alpha-Gal A with ERT as well as treating the various symptoms and complications [18]. Migalastat is an oral pharmacological chaperone that binds to and stabilizes specific mutant forms of GLA, thereby facilitating proper trafficking of the enzyme to lysosomes and increasing enzyme activity. Therefore, it can be used instead of ERT in patients with amenable genetic variants that allow a substantial increase in enzyme activity. ERT candidates should be evaluated for the presence of certain GLA variants to determine if they may be potential candidates for migalastat therapy. Although ERT may reduce neuropathic pain, its beneficial effects on the severity and/or progression of other disease manifestations are less clear. Hypothetically ERT may reduce tissue accumulation of Gb3 in endothelial cells in the heart, skin, and in most cell types in the kidneys. Therefore, if ERT is used at the early stages of kidney disease, it may slow renal function decline. ERT does not significantly reduce the risk of stroke or heart disease. The short-term and long-term results of kidney transplantation for FD are comparable with transplantation for other causes of ESRD. As with other causes of ESRD, survival with transplantation is superior to that observed with dialysis. Thus, transplantation should be the treatment of choice for ESRD due to FD. A clinically significant Fabry nephropathy does not recur in the transplanted kidney, although some recipients may develop Gb3 deposition that typically does not compromise graft function [19].

Survival is substantially reduced in males with classic FD. Before dialysis, such patients usually died in the fourth decade of life. With the availability of dialysis, life expectancy has increased to the fifth decade [20]. The primary cause of death in patients with FD is from cardiac complications [20].

\section{Conclusions}

FD is an X-linked lysosomal storage disease affecting multiple organs. Clinical manifestations are nonspecific and heterogeneous. The diagnosis is established by a low leukocyte alpha-Gal A activity and a variety of mutations in the GLA gene. LM examination of FD shows foamy vacuolization in glomerular cells, tubular epithelial cells, and vascular cells. EM examination shows myelin or zebra bodies. Although renal biopsy and histopathological findings are not needed for the diagnosis of FD, they are a necessary tool to monitor the renal progression and to evaluate the efficiency of ERT. Patients with ESRD secondary to FD should be referred to renal transplant centers, as renal transplantation improves their prognosis.

\section{Additional Information \\ Disclosures}

Human subjects: Consent was obtained by all participants in this study. Conflicts of interest: In compliance with the ICMJE uniform disclosure form, all authors declare the following: Payment/services info: All authors have declared that no financial support was received from any organization for the submitted work. Financial relationships: All authors have declared that they have no financial relationships at present or within the previous three years with any organizations that might have an interest in the submitted work. Other relationships: All authors have declared that there are no other relationships or activities that could appear to have influenced the submitted work.

\section{Acknowledgements}

The views expressed in this publication represent those of the authors and do not necessarily represent the official views of HCA healthcare or any of its affiliated entities.

\section{References}

1. Bishop DF, Kornreich R, Desnick RJ: Structural organization of the human alpha-galactosidase a gene: further evidence for the absence of a 3' untranslated region. Proc Natl Acad Sci U S A. 1988, 85:3903-7. 10.1073/pnas.85.11.3903

2. El-Abassi R, Singhal D, England JD: Fabry's disease. Review J Neurol Sci. 2014, 344:5-19. 10.1016/j.jns.2014.06.029

3. Spada M, Pagliardini S, Yasuda M, et al.: High incidence of later-onset Fabry disease revealed by newborn screening. Am J Hum Genet. 2006, 79:31-40. 10.1086/504601

4. Aerts JM, Groener JE, Kuiper S, et al.: Elevated globotriaosylsphingosine is a hallmark of Fabry disease . Proc Natl Acad Sci U S A. 2008, 105:2812-7. 10.1073/pnas.0712309105

5. Sanchez-Niño, Sanz AB, Carrasco S, et al.: Globotriaosylsphingosine actions on human glomerular podocytes: implications for Fabry nephropathy. Ephrol Dial Transplant. 2011, 26:1797-802. 10.1093/ndt/gfq306

6. Schaefer E, Mehta A, Gal A: Genotype and phenotype in Fabry disease: analysis of the Fabry Outcome Survey. Acta Paediatr Suppl. 2005, 94: 87-92. 10.1111/j.1651-2227.2005.tb02119.x 
7. Houge G, Skarbøvik AJ: [Fabry disease--a diagnostic and therapeutic challenge] . Tidsskr Nor Laegeforen. 2005, 125:1004-6.

8. Arends M, Wanner C, Hughes D, et al.: Characterization of classical and nonclassical Fabry disease: a multicenter study. J Am Soc Nephrol. 2017, 28:1631-41. 10.1681/ASN.2016090964

9. Desnick RJ, Brady R, Barranger J: Fabry disease, an under-recognized multisystemic disorder: expert recommendations for diagnosis, management, and enzyme replacement therapy. Ann Intern Med. 2003, 138:338-46. 10.7326/0003-4819-138-4-200302180-00014

10. Wilcox WR, Oliveira JP, Hopkin RJ: Females with Fabry disease frequently have major organ involvement: lessons from the Fabry Registry. Mol Genet Metab. 2008, 93:112-28. 10.1016/j.ymgme.2007.09.013

11. Hagège AA, Caudron E, Damy T, et al.: Screening patients with hypertrophic cardiomyopathy for Fabry disease using a filter-paper test: the FOCUS study. Heart. 2011, 97:131-6. 10.1136/hrt.2010.200188

12. Branton MH, Schiffmann R, Sabnis SG, et al.: Natural history of Fabry renal disease: influence of alphagalactosidase A activity and genetic mutations on clinical course. Medicine (Baltimore). 2002, 81:122-38. 10.1097/00005792-200203000-00003

13. Tanaka M, Ohashi T, Kobayashi M, et al.: Identification of Fabry's disease by the screening of alphagalactosidase A activity in male and female hemodialysis patients. Clin Nephrol. 2005, 64:281-7. $10.5414 /$ cnp64281

14. Najafian B, Svarstad E, Bostad L, Gubler MC, Tøndel C, Whitley C. Mauer M: Progressive podocyte injury and globotriaosylceramide (GL-3) accumulation in young patients with Fabry disease. Kidney Int. 2011, 79:663670. 10.1038/ki.2010.484

15. Fall B, Scott CR, Mauer M, et al.: Urinary podocyte loss is increased in patients with Fabry disease and correlates with clinical severity of Fabry nephropathy. PLoS One. 2016, 11:e0168346. 10.1371/journal.pone.0168346

16. Branton M, Schiffmann R, Sabnis S, et al.: Natural history of Fabry renal disease: influence of agalactosidase activity and genetic mutations on clinical course. Medicine (Baltimore). 2002, 81:122-38. 10.1097/00005792-200203000-00003

17. Alroy J, Sabnis S, Kopp JB: Renal pathology in Fabry disease. J Am Soc Nephrol. 2002, 13:134-138. 10.1097/01.ASN.0000016684.07368.75

18. Schiffmann R, Murray GJ, Treco D, et al.: Infusion of alpha-galactosidase A reduces tissue globotriaosylceramide storage in patients with Fabry disease. Proc Natl Acad Sci U S A. 2000, 97:365-370. 10.1073/pnas.97.1.365

19. Ojo A, Meier-Kriesche HU, Friedman G, Hanson J, Cibrik D, Leichtman A, Kaplan B: Excellent outcome of renal transplantation in patients with Fabry's disease. Transplantation. 2000, 69:2337-9. 10.1097/00007890200006150-00020

20. Mehta A, Clarke JT, Giugliani R, Elliott P, Linhart A, Beck M, Sunder-Plassmann G: Natural course of Fabry disease: changing pattern of causes of death in FOS - Fabry Outcome Survey. J Med Genet. 2009, 46:548552. 10.1136/jmg.2008.065904 